\title{
Prognostic value of microvessel density in cervical cancer
}

\author{
Xiaoli Hu, Hailing Liu, Miaomiao Ye and Xuegiong Zhu* (i)
}

\begin{abstract}
Background: Several epidemiological researches have indicated that microvessel density (MVD), reflecting angiogenesis, was a negatively prognostic factor of cervical cancer. However, the results were inconsistent. Therefore, we performed a meta-analysis to evaluate the association between microvessel density and the survival probability of patients with cervical cancer.

Method: There was a comprehensive search of the PubMed, EMBASE and Cochrane databases up to August 31, 2017. Based on a fixed-effects or random-effects model, the hazard ratio (HR) and 95\% confidence intervals (Cls) were calculated from researches on overall survival (OS) and disease-free survival (DFS).

Result: Totally, we included 13 observational researches, involving 1097 patients with cervical cancer. The results showed that high level of microvessel density was negatively correlated with OS ( $H R=1.79,95 \%$ Cls $1.31-2.44$, $\left.P^{2}=60.7 \%, P=0.003\right)$ and DFS (HR=1.47, 95\% Cls $\left.1.13-1.80, P^{2}=0 \%, P=0.423\right)$ of cervical cancer patients. In subgroup analysis, high counts of MVD were significantly associated with a poor survival (including OS and DFS) of the patients detected by anti-factor VIII antibodies or in European origin.
\end{abstract}

Conclusion: The present meta-analysis indicated that survival with high level of MVD was significant poorer than with low MVD in cervical cancer patient. Standardization of MVD assessment is needed.

Keywords: Microvessel density, Survival, Cervical neoplasia, Meta-analysis

\section{Background}

Cervical cancer is the third most frequent gynecological neoplasms worldwide and one of the leading causes of cancer-related death among women in developing countries [1]. This disease is responsible for approximately 265,000 deaths annually in the world, $87 \%$ occurring in low-income countries [2]. Although independent prognostic factors such as lymph node status, tumor size, pathologic grading of tumor and International Federation of Gynecology and Obstetrics (FIGO) stage contribute to a better comprehension of the disease progression $[3,4]$. However, such factors couldn't predict individual clinical outcome absolutely in cervical cancer. Thus, there need more prognostic markers to further improve predictive accuracy.

*Correspondence: zjwzzxq@163.com

Department of Obstetrics and Gynecology, The Second Affiliated Hospital of Wenzhou Medical University, Wenzhou 325000, Zhejiang, China
Angiogenesis have been reported to play a crucial role in the growth, metastasis and progression of various types of cancers like breast cancer and renal cell carcinoma $[5,6]$. When a tumor exceeds the size of $1 \mathrm{~mm}$, its further growth needs angiogenesis, which can form new blood vessels and further lead to tumor metastasis. There are a variety of biomarkers to quantify intratumoral angiogenesis, including vascular endothelial growth factor (VEGF), basic fibroblast growth factor (bFGF) and microvessel density (MVD).

At present, MVD assessment is the most common method to evaluate intratumor angiogenesis in cancer. In early 1990s, MVD was firstly introduced as an indicator by Weidner et al. [7] to assess the microvessels density in patients with invasive breast cancer. Additionally, the most commonly used antibodies for microvessel staining are CD31, CD34, CD105 (Endoglin) and anti-factor VIII (Von Willebrand Factor). Over the past two decades, 
some studies had reported MVD as a prognosis factor in various tumors such as gastric carcinoma [8].

However, the value of microvessel density as a prognostic indicator of cervical cancer was controversial. Several researches demonstrated that the expression of MVD significantly associated with poor overall survival (OS) or progression free survival (PFS) for cervical cancer $[9,10]$. But some studies were unable to indicate a significant relationship between MVD and poor survival in cervical cancer patients [11, 12].

Therefore, we conducted a meta-analysis in order to evaluate the prognostic value of microvessel density in patients with cervical carcinoma. Meanwhile, this study may help to provide a valuable prognostic indicator and guide the management of the cervical cancer patients in the future.

\section{Methods}

\section{Search strategy}

Two investigators conducted a comprehensive search in electronic databases of PubMed, EMBASE and Cochrane library for relevant researches up to 31 August 2017. The following Medical Subject Heading terms and keywords were used: ("cervical cancer" or "cervical tumor" or "cervical tumour" or "cervical malignance" or "cervical carcinoma" or "uterine cervical neoplasms" or "cervical neoplasm") and ("microvessel density" or "MVD" or "angiogenesis" or "neovascularization") and ("prognosis" or "outcome" or "survival" or "prognostic") with no restrictions. Meanwhile, we also performed a manual search of references cited in the retrieved studies and published reviews.

\section{Selection criteria}

To be eligible, researches must be consistent with the following inclusion criteria: (1) All included patients with cervical carcinoma diagnosed by the pathological results; (2) reported the association between MVD and survival outcomes, such as overall survival (OS) and disease free survival (DFS); (3) papers were restricted to human studies published as full-length articles in English. Exclusion criteria were (1) reviews, letters, case reports, or editorial comments; (2) duplicate publications; (3) full text unavailable; (4) insufficient data for calculating the hazard ratios (HRs) and 95\% confidence intervals (CIs).

The candidate studies were identified by two independent reviewers according to the titles and abstracts to exclude irrelevant studies. Then full texts of the remaining researches were scanned carefully to decide whether to include the studies, and any different opinion was resolved through discussion. Multivariate data were the priority choice when both multivariate and univariate data were offered. However, we also accepted univariate data when no multivariate results were provided.

\section{Data extraction and quality assessment}

The following information were extracted carefully from all including studies by two authors by means of a standardized data table which included following items: the first author; the year of publication; the location of study; the number of included patients; the age range of participants; FIGO stage; the antibody to assess MVD; the duration of follow up; the cutoff value of MVD (usually with median MVD as cutoff); the types of survival analyses; HRs and 95\% CI for overall survival/diseasefree survival; the result of the study. The result for every single study was marked "positive" when higher MVD predicted poorer survival and "negative" when higher MVD did not indicate lower survival rate or even once supported a better survival.

Quality assessment of the including studies was evaluated by two investigators independently using the 9-star Newcastle-Ottawa Scale (NOS) [13]. According to the scoring system, we defined the research quality as high with scores which were equal to or greater than 7.

\section{Statistical analysis}

The prognostic efficiency of MVD on cervical carcinoma was calculated by using the HRs and 95\% CIs. When the effect values couldn't be provided directly by the study, we calculated HR value and 95\% corresponding CIs in the Kaplan-Meier curve at particular time points using the methods introduced by Parmar et al. [14]. An observed HR $>1$ indicated a bad prognosis in cervical cancer patient with the high MVD. Statistical heterogeneity from all the publications was tested by Cochran's $Q$ test and Higgins I-squared statistics [15]. Meanwhile, a fixed-effects model was adopted to assess the pooled value when $I^{2}<50 \%$ and $P>0.10$ which indicated that no obvious heterogeneity was found. Otherwise, a randomeffects model was applied. All statistical analyses were performed with STATA 11.0 (STATA Corp, College Station, Texas).

In addition, Subgroup analyses were conducted to calculate the potential source of heterogeneity according to geographical regions and antibodies for detecting MVD. A sensitivity analysis evaluating the consistency of the combined outcomes was adopted. The possible publication bias was assessed by Begg's tests [16]. All P-value were two-tailed and statistical significance was obvious as $P<0.05$. 


\section{Results}

Literature search

At the beginning, there were a total of 580 articles identified from three databases (262 from PubMed, 298 from EMBASE, 18 from Cochrane databases) according to the inclusion criteria. After screening the title, abstract and key words, 427 articles were deleted when met duplication. Followed by excluded researches which were obvious irrelevant, or didn't meet the inclusion criteria, we adopted a number of 13 independent and observational studies involving 1097 patients [911, 17-26]. The flow chart presented in Fig. 1 showed the study selection process in detail.

\section{Study characteristics}

Table 1 presented the main characteristics of the 13 studies included in the meta-analysis. These researches were published between 1995 and 2014, and the sample sizes ranged from 30 to 173. As for the region, three studies were conducted from America [9, 21, 24], seven in Europe $[10,17,18,22,23,25,26]$ and three in Asian country $[11,19,20]$. In addition, there were four different antibodies enrolled in the included studies to assess the microvessel density. Anti-factor VIII antibody was used in six studies $[10,18-20,22,26]$, CD 31 was conducted in four studies [17, 21, 23, 24], CD 34 was in two articles $[9,11]$, CD 105 was applied in two studies [21, 23], respectively. And all of the protein-levels of antibody were detected by immunohistochemistry (IHC). Among

\section{Records identified through database searching PubMed ( $\mathrm{n}=262)$, Embase ( $\mathrm{n}$ $=298)$ and Cochrane databases $(n=18)$}

Additional records identified through other sources $(n=2)$

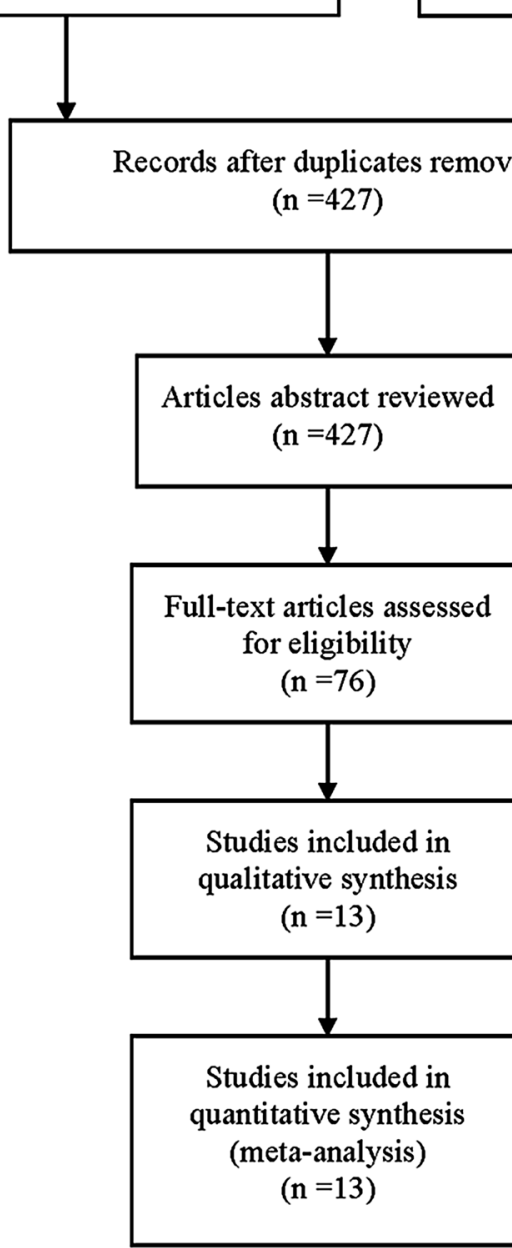

Fig. 1 Flow diagram for study selection 


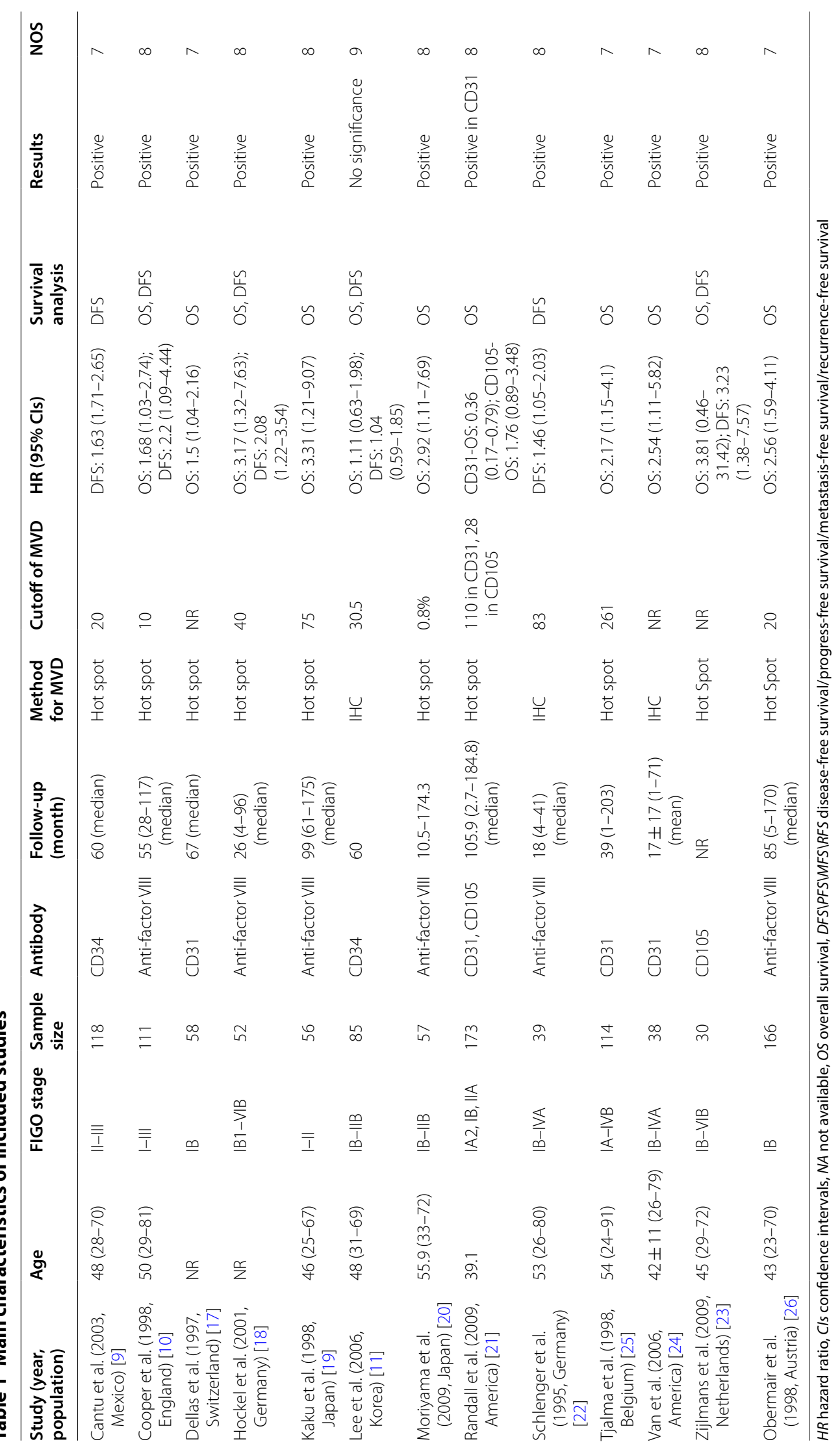


them, MVD count in ten studies was performed by hotspot method which was introduced by Weidner et al. [7], while the hotspot method was not mentioned in other three articles. Thus, 14 researches (from 13 articles) involving 1097 patients were available for this meta-analysis. We included 11 studies which gave a description of the association between OS and MVD, and six researches involved other outcomes such as DFS. Otherwise, we defined the study as ' -1 ' and ' -2 ' if more than one outcome or antibody were applied in the same study [27, 28]. Meanwhile, the quality of included researches were assessed by using the Newcastle-Ottawa Scale and found to range from 7 stars to 9 stars, showing that the studies were in high quality.

\section{Association of MVD and OS of cervical cancer}

The pooled HR for the 11 studies assessing the association between MVD and cervical cancer with OS was 1.7 (95\% CIs 1.31-2.44, random-effects, Fig. 2), suggesting that high MVD level was associated with a poor prognosis of overall survival in cervical cancer patients. Since the heterogeneity among studies was significant
$\left(I^{2}=60.7 \%, \quad P=0.003\right), \quad$ random-effects model was applied for statistical analysis. Meanwhile, subgroup meta-analysis according types of antibodies and population was conducted to evaluate the possible source of heterogeneity among these studies (Figs. 3 and 4).

In the subgroup analysis by different antibodies, the prognostic value of MVD for OS was significant in the "anti-factor VIII" subgroup ( $\mathrm{HR}=2.34,95 \%$ CIs $1.75-$ $3.12, I^{2}=0 \%, \mathrm{n}=5$ ), while there was not statistically significant association in "CD31" subgroup ( $\mathrm{HR}=1.33$, 95\% CIs $0.65-2.72, I^{2}=81.2 \%, \mathrm{n}=4$ ), "CD34" subgroup $(\mathrm{HR}=1.11,95 \% \mathrm{CIs} 0.63-1.97, \mathrm{n}=1)$ and "CD105" subgroup $\left(\mathrm{HR}=1.89,95 \%\right.$ CIs $\left.0.99-3.62, I^{2}=0 \%, \mathrm{n}=2\right)$.

There was another subgroup about source regions of included studies, a pooled HR was 1.93 (95\% CIs 1.53$2.44, I^{2}=4.8 \%, \mathrm{n}=6$ ) in Europe population, indicating a significantly poorer survival in cervical cancer patients with higher MVD in European countries. However, MVD level was not significantly associated with OS in Asia $\left(\mathrm{HR}=2.01,95 \%\right.$ CIs $\left.0.94-4.31, I^{2}=60.1 \%, \mathrm{n}=3\right)$ and American locations ( $\mathrm{HR}=1.17,95 \%$ CIs $0.37-3.69$, $I^{2}=85.5 \%, \mathrm{n}=3$ ).

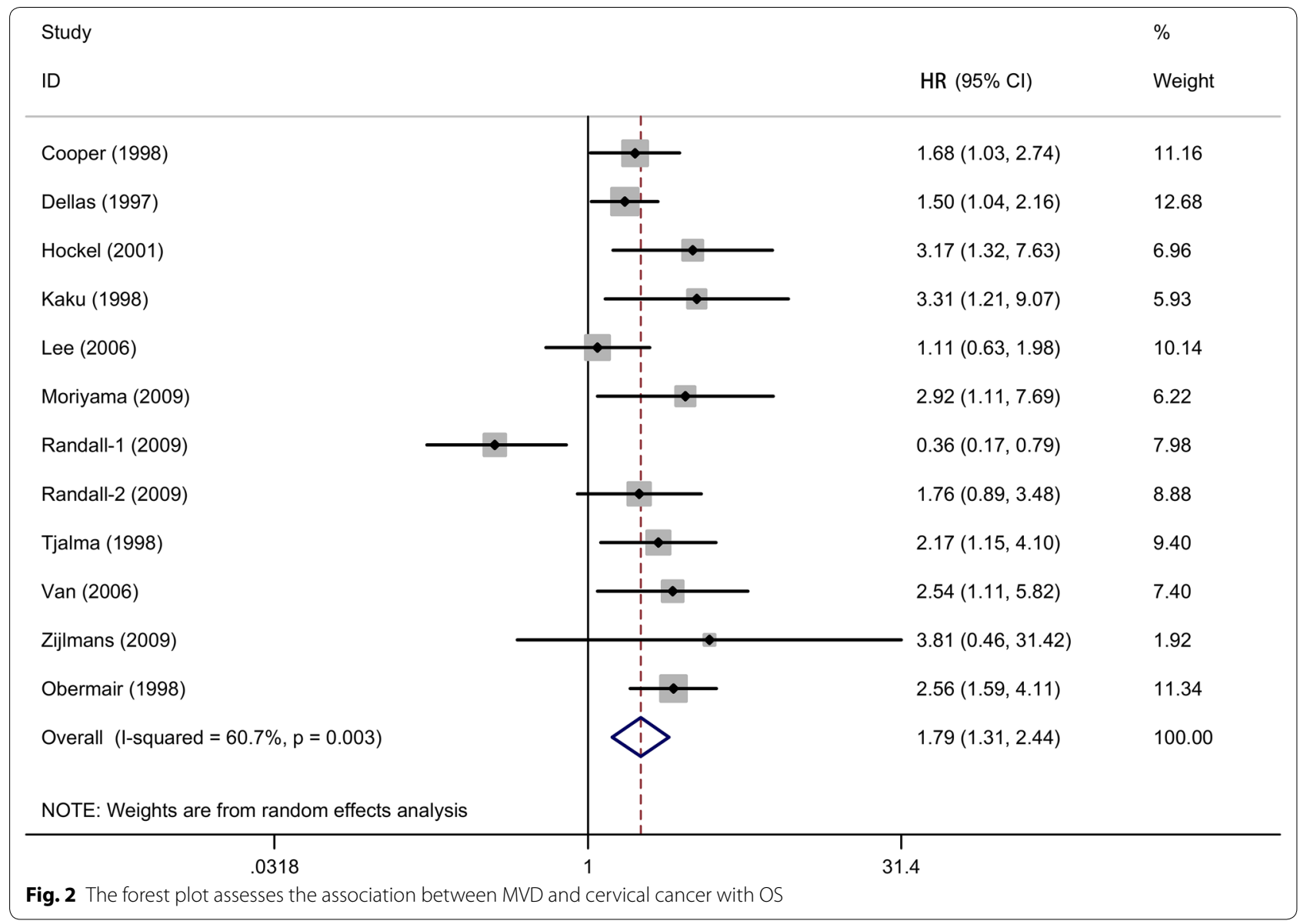




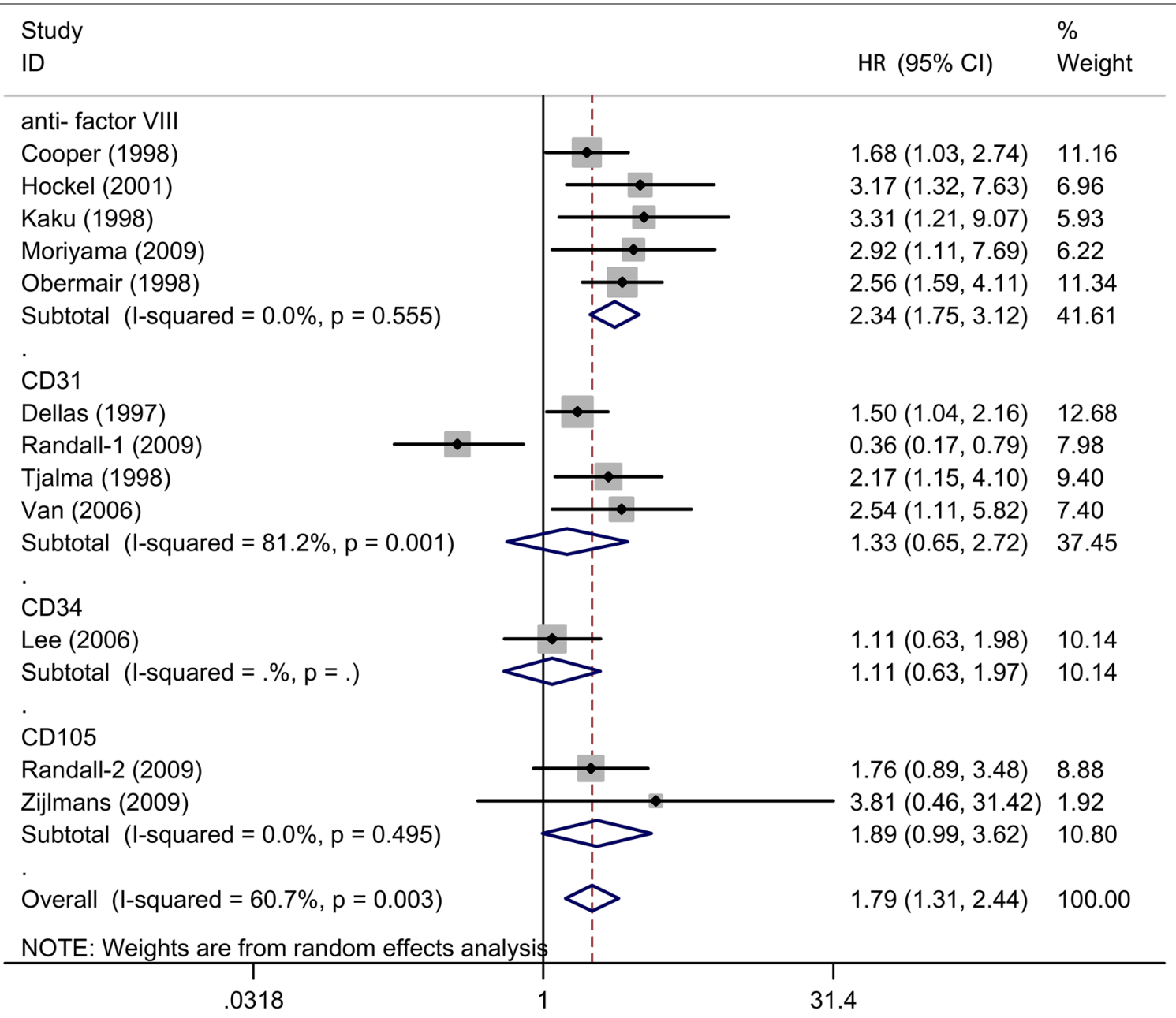

Fig. 3 Subgroup analysis of association between count of MVD and prognosis of cervical cancer with OS detected by different antibodies

\section{Association of MVD and DFS of cervical cancer}

We analyzed the relationship between the level of MVD and DFS among cervical cancer patients. There was no heterogeneity of data $\left(I^{2}=0 \%\right)$, in which a fixed-effect model was selected to assess the pooled outcome (Fig. 5). As a result, MVD level was associated with a worse DFS of cervical cancer patients ( $\mathrm{HR}=1.47,95 \%$ CIs $1.13-1.80$, $\mathrm{n}=6$ ).

Furthermore, subgroup analyses based on antibody and region were used to explore the influencing factors which may impacted the overall outcomes (Figs. 6 and 7). Divided by different immunohistochemical biomarkers among the subgroups, "anti-factor VIII" antibody $\left(\mathrm{HR}=1.60,95 \%\right.$ CIs 1.16-2.03, $\left.I^{2}=0, \mathrm{n}=3\right)$ showed the significantly negative association between MVD and DFS among cervical cancer patients, but not in "CD34" subgroup (HR $=1.23,95 \%$ CIs $\left.0.71-1.75, I^{2}=8.4 \%, \mathrm{n}=2\right)$.

In addition, the included studies were stratified into the three regional distribution of patients (Europe, Asia and
America). Negative effected of MVD on DFS in European countries were found in patients with cervical cancer $\left(\mathrm{HR}=1.63,95 \%\right.$ CIs $\left.1.20-2.06, I^{2}=0 \%, \mathrm{n}=4\right)$, but not in Asia location $\left(\mathrm{HR}=1.47,95 \%\right.$ CIs $1.13-1.80, I^{2}=0 \%$, $\mathrm{n}=2$ ).

\section{Sensitivity analysis}

In sensitivity analysis, the leave-one-out method was applied to assess the stability of the pooled outcomes. Eligible studies were sequentially removed one by one to evaluate the influence of each included study on the overall HR. After leaving out any single study, statistical significance of the OS or DFS did not change, suggesting no individual study had excessive influence of the association of MVD and cervical cancer (Fig. 8a, b).

\section{Publication bias}

The presence of publication bias for the overall relationship between MVD level and the prognosis of cervical 


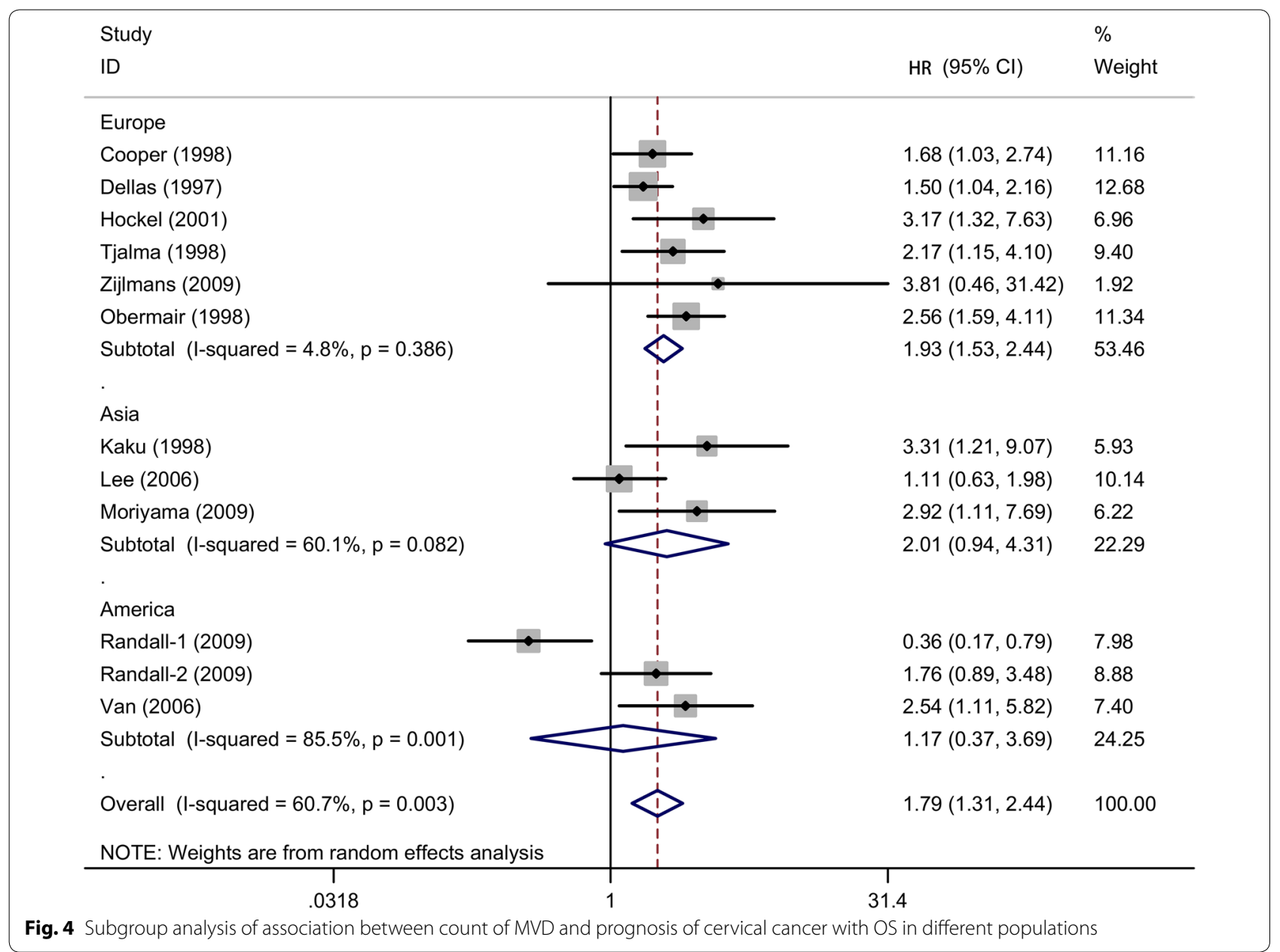

was calculated by using the Begg's rank correlation test $(P=0.15$ for OS; $P=0.238$ for DFS). Funnel plots were graphically symmetric, indicating that there was no significant publication bias among the included articles (Fig. 9a, b).

\section{Discussion}

A number of studies have showed that MVD played the potential role as prognostic biomarker for many cancers. In our present meta-analysis, we confirmed that high counts of MVD, a marker of angiogenesis, was significantly associated with worse prognosis in patients with cervical cancer. Moreover, results were also conducted in subgroup analyses for patients detected by different antibodies or in various countries. Although some modest bias cannot be excluded, this was the first meta-analysis of published articles to assess the relationship between MVD count and prognosis in cervical cancer.

Between-study heterogeneity was significant in our meta-analysis for OS $\left(I^{2}=60.7 \%\right)$. However, there was no heterogeneity for DFS $\left(I^{2}=0 \%\right)$. We tried to reduce the variability by screening the researches using the same standard, which was to divide studies into different subgroups, such as the regional distributions and staining markers. However, the heterogeneity could not be eliminated in general. But the heterogeneity had decreased in some subgroups such as the Europe group $\left(I^{2}=4.8 \%\right.$ and $11.8 \%)$ and the group using anti-factor VIII as biomarkers on OS $\left(I^{2}=0 \%\right)$ for the MVD group. These results showed that all the different factors played important roles in the generation of heterogeneity which couldn't be eliminated at the same time.

Obviously, our study showed that the selection of antibody as a biomarker for MVD assessment played an important role for conclusion. There were eight studies in our meta-analysis using factor VIII as an endothelial biomarker, eight studies using other antibodies such as CD31, CD34 and CD105. We have established that the counts of MVD assessed by factor VIII were significantly related with the poor outcomes of cervical cancer, including OS and DFS. However, there was no statistical significance in the association between the levels of MVD 


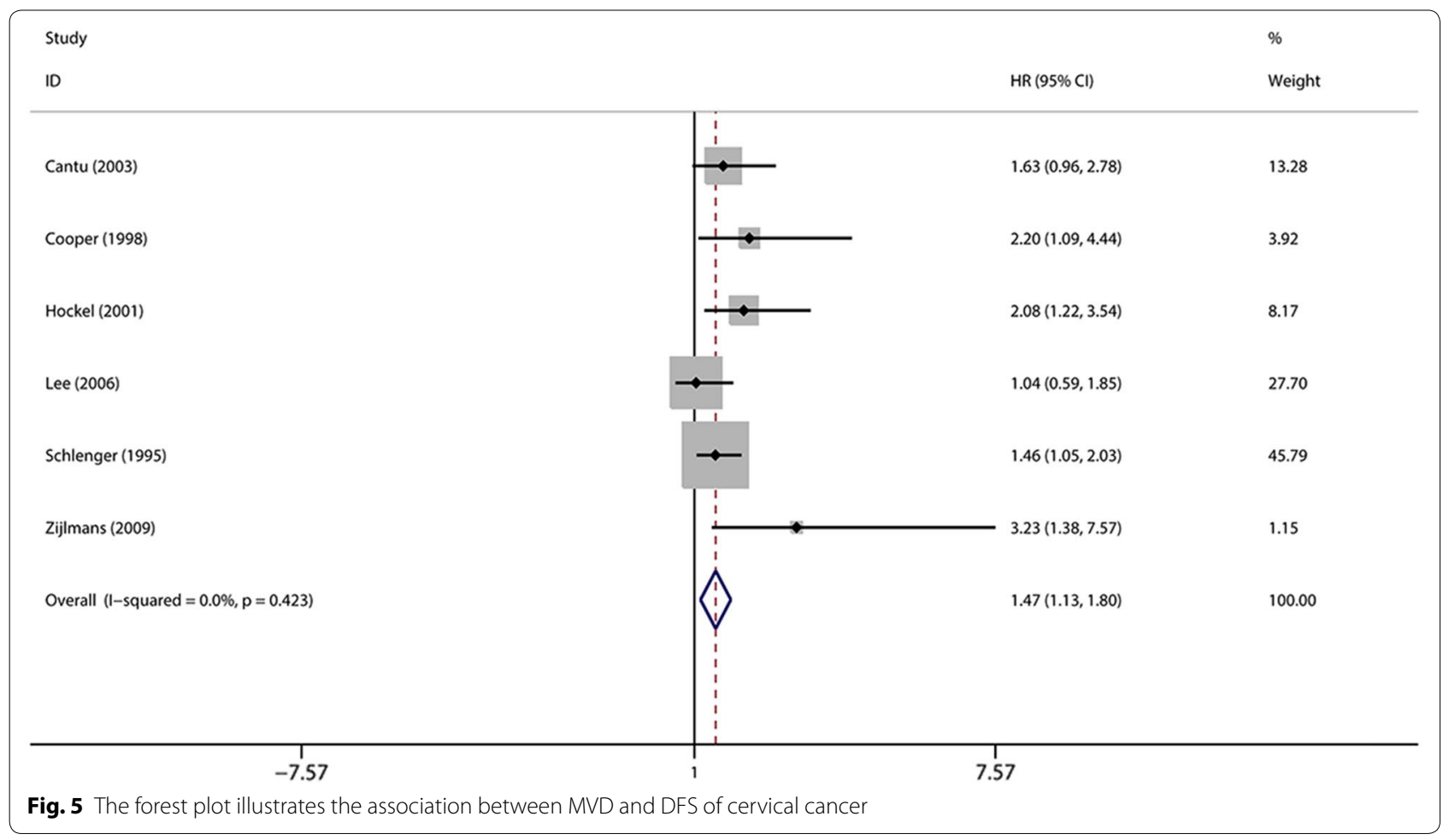

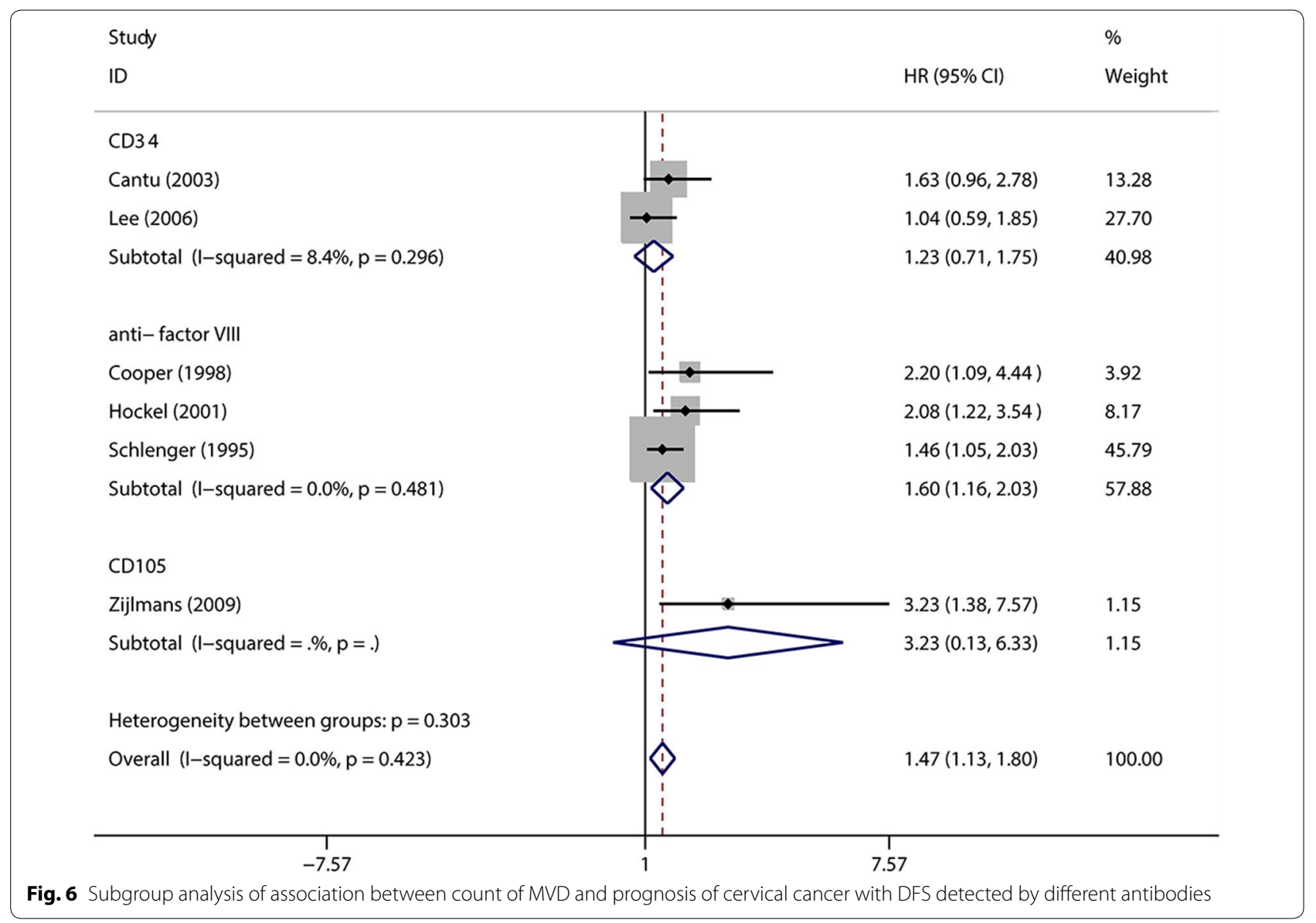




\begin{tabular}{|c|c|c|}
\hline Study & & $\%$ \\
\hline ID & $\mathrm{HR}(95 \% \mathrm{Cl})$ & Weight \\
\hline America & & \\
\hline Cantu (2003) & $1.63(0.96,2.78)$ & 13.28 \\
\hline Subtotal (I-squared $=. \%, p=)$. & $1.63(0.72,2.54)$ & 13.28 \\
\hline \multicolumn{3}{|l|}{ Europe } \\
\hline Cooper (1998) & $2.20(1.09,4.44)$ & 3.92 \\
\hline Hockel (2001) & $2.08(1.22,3.54)$ & 8.17 \\
\hline Schlenger (1995) & $1.46(1.05,2.03)$ & 45.79 \\
\hline Zijlmans (2009) & $3.23(1.38,7.57)$ & 1.15 \\
\hline Subtotal $(I-$ squared $=0.0 \%, p=0.473)$ & $1.63(1.20,2.06)$ & 59.02 \\
\hline \multicolumn{3}{|l|}{ Asia } \\
\hline Lee (2006) & $1.04(0.59,1.85)$ & 27.70 \\
\hline Subtotal $(1-$ squared $=. \%, p=)$. & $1.04(0.41,1.67)$ & 27.70 \\
\hline Heterogeneity between groups: $p=0.296$ & & \\
\hline Overall $(I-$ squared $=0.0 \%, p=0.423)$ & $1.47(1.13,1.80)$ & 100.00 \\
\hline $\begin{array}{c}1 \\
-7.57 \\
\end{array}$ & $\begin{array}{l}1 \\
7.57\end{array}$ & \\
\hline
\end{tabular}
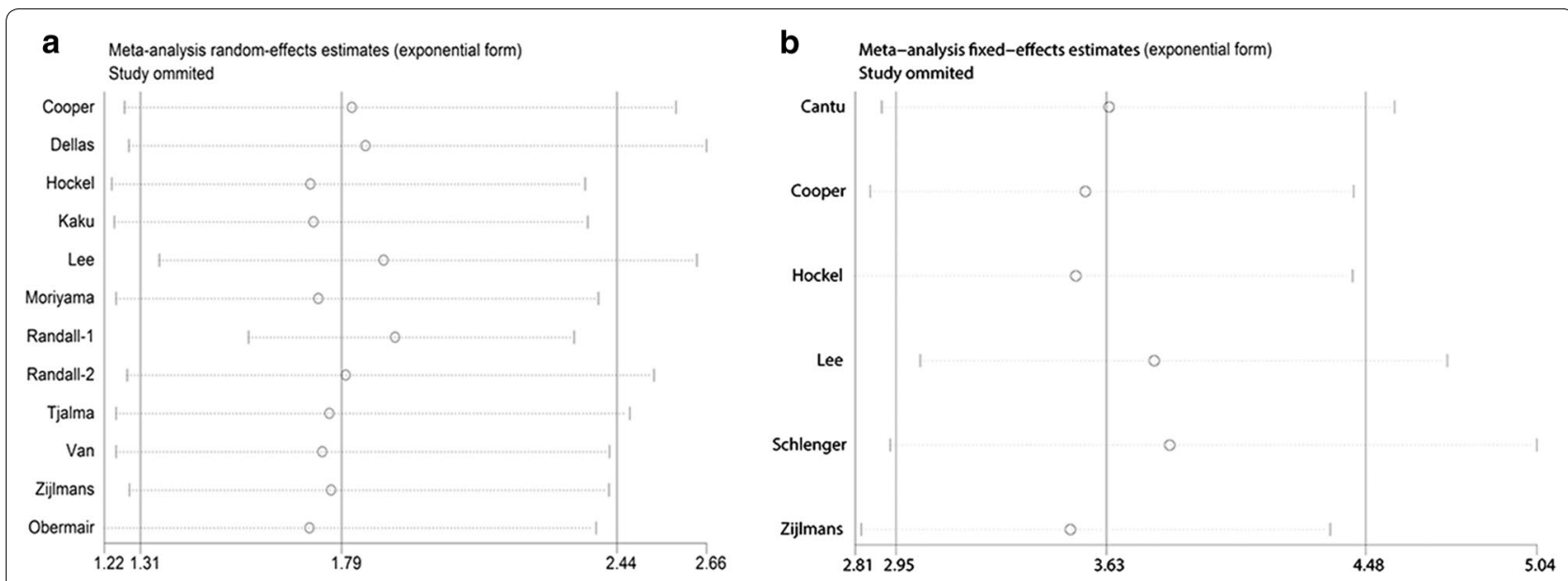

Fig. 8 Sensitivity analysis to assess the stability of the pooled outcomes between count of MVD and prognosis of cervical cancer. $\mathbf{a}$ OS; $\mathbf{b}$ DFS 

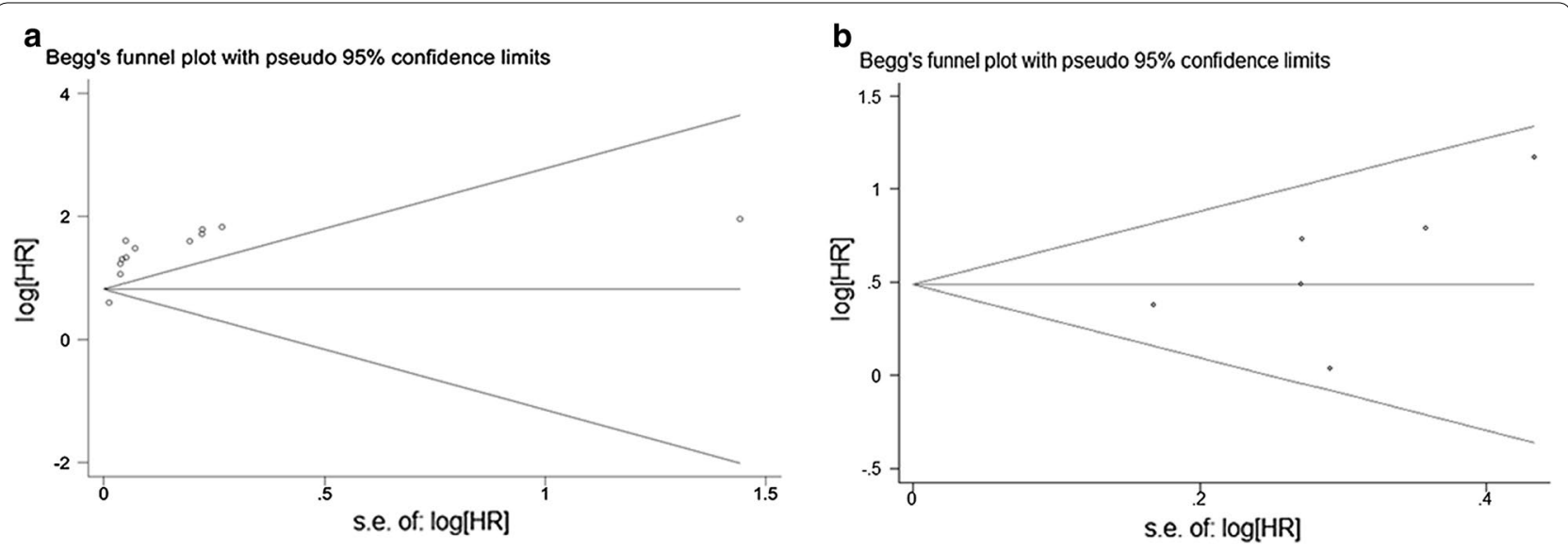

Fig. 9 The presence of publication bias for the overall relationship between MVD level and the prognosis of cervical cancer. a OS; $\mathbf{b}$ DFS

assessed by anti-CD31, CD34 or CD105 with the prognosis of cervical cancer patients for OS or DFS. Weider et al. [7] chose an antibody against factor VIII-related antigen to mark mainly the endothelia of mature vessels. This biomarker was still the most commonly used in the related studies about microvessel density, however factor VIII did not express in all endothelial cells. And factor VIII also expressed in lymphatic endothelium and platelets, which would result in cross-reactivity with non-endothelium [29]. At present, for these antibodies expressed in lymphatic endothelium and platelets leading to appropriate marker for MVD in tumor has not been established. Recently, other antibodies were used to assess the counts of MVD in cervical cancer [30,31], such as CD31 (also known as platelet endothelial cell adhesion molecule), CD34 and CD105 (also known as Endoglin). It indicated that CD34 had an improved sensitivity and specificity than factor VIII for endothelial cells activated by regional tumor angiogenesis [32]. Uzzan et al. [27] found that the microvessel counting evaluated by antiCD31 or anti-CD34 were approximately $30 \%$ higher than factor VIII. Therefore, we considered that no statistical significance of relationship between counts of MVD assessed by other biomarkers including CD31, CD34 and CD105, and prognosis of cervical cancer patients was limited by a few number of related studies. Thus, more high-quality studies concerning the MVD detecting and MVD count should be acquired in future. Meanwhile, we found that there were more researches about the relation between MVD and prognosis of cervical cancer patients in Europe group than in Asia and America. When stratified by geographical area, our subgroup analysis indicated that patients with cervical cancer who had a higher level of MVD would had the poorer survival in Europe countries, while the results could not be verified in Asia and America. It was probably because of the insufficient quantity in above-mentioned two regions.

In addition, several limitations of this meta-analysis should also be discussed. First of all, the choice of cutoff values for high MVD varied among the studies, some included articles mainly used median level, while others applied mean or even an inaccurate bound. These differences were responsible for the difficulty in determining a standard cut off value in clinical practice. Therefore, future researches should aim to standardize MVD assessment method. Secondly, for HRs couldn't be provided directly or calculated from the data in some studies, we need to extract the data from survival curve graphs. Furthermore, it is inevitable that the patient's baseline status in included studies were different, such as age, menopausal status, tumor type, tumor size, lymph node status, the immunohistochemical marker for MVD detecting and duration of follow-up. Finally, all the included studies in our meta-analysis were retrospective observational researches, more prone to bias than randomized controlled trails.

\section{Conclusions}

To conclude, despite above-mentioned limitations, our meta-analysis strongly showed a poor survival of high counts of MVD in patients with cervical cancer, including OS and DFS, respectively. Moreover, these MVD-related biomarkers could be further used in the prognosis prediction of cervical cancer in clinical practice. However, future basic researches and randomized controlled studies with large samples are needed to conduct the prognostic value of MVD for patients with cervical cancer. 


\section{Abbreviations}

MVD: microvessel density; HR: hazard ratio; Cls: confidence intervals; OS: overall survival; DFS: disease-free survival; FIGO: International Federation of Gynecology and Obstetrics; VEGF: vascular endothelial growth factor; bFGF: basic fibroblast growth factor; NOS: the 9-star Newcastle-Ottawa Scale; IHC: immunohistochemistry; NA: not available.

\section{Authors' contributions}

$\mathrm{XLH}$ and $\mathrm{XQZ}$ designed the study; XLH and $\mathrm{HL}$ searched and selected the articles, conducted the data extraction and did the statistical analysis; XLH and MMY wrote the manuscript. All authors read and approved the final manuscript.

\section{Acknowledgements}

Not applicable.

\section{Competing interests}

The authors declare that they have no competing interests.

\section{Availability of data and materials}

The data and material of this research are included in this published article.

\section{Consent for publication}

Not applicable.

\section{Ethics approval and consent to participate}

This study was approved by the ethics committee at the Second Hospital of Wenzhou Medical University.

\section{Funding}

This work was supported by grants from Center for Uterine Cancer Diagnosis \& Therapy Research of Zhejiang Province and Key Lab of Wenzhou CityGynecological Oncology (No. ZD201603).

\section{Publisher's Note}

Springer Nature remains neutral with regard to jurisdictional claims in published maps and institutional affiliations.

Received: 18 January 2018 Accepted: 24 September 2018 Published online: 03 October 2018

\section{References}

1. Siegel RL, Miller KD, Jemal A. Cancer statistics, 2018. CA Cancer J Clin. 2018;68(1):7-30.

2. Kress CM, Sharling L, Owen-Smith AA, Desalegn D, Blumberg HM, Goedken J. Knowledge, attitudes, and practices regarding cervical cancer and screening among Ethiopian health care workers. Int J Womens Health. 2015:7:765-72.

3. Intaraphet S, Kasatpibal N, Siriaunkgul S, Sogaard M, Patumanond J, Khunamornpong S, Chandacham A, Suprasert P. Prognostic impact of histology in patients with cervical squamous cell carcinoma, adenocarcinoma and small cell neuroendocrine carcinoma. Asian Pac J Cancer Prev. 2013;14(9):5355-60

4. Horn LC, Bilek K, Fischer U, Einenkel J, Hentschel B. A cut-off value of $2 \mathrm{~cm}$ in tumor size is of prognostic value in surgically treated FIGO stage IB cervical cancer. Gynecol Oncol. 2014;134(1):42-6.

5. Leek RD. The prognostic role of angiogenesis in breast cancer. Anticancer Res. 2001;21(6B):4325-31.

6. Jilaveanu LB, Puligandla M, Weiss SA, Wang XV, Zito C, Flaherty KT, Boeke M, Neumeister V, Camp RL, Adeniran A, et al. Tumor microvessel density as a prognostic marker in high-risk renal cell carcinoma patients treated on ECOG-ACRIN E2805. Clin Cancer Res. 2018;24(1):217-23.

7. Weidner N, Semple JP, Welch WR, Folkman J. Tumor angiogenesis and metastasis - correlation in invasive breast carcinoma. N Engl J Med. 1991;324(1):1-8.

8. Cao F, Hu YW, Li P, Liu Y, Wang K, Ma L, Li PF, Ni CR, Ding HZ. Lymphangiogenic and angiogenic microvessel density in chinese patients with gastric carcinoma: correlation with clinicopathologic parameters and prognosis. Asian Pac J Cancer Prev. 2013;14(8):4549-52.

9. Cantu De Leon D, Lopez-Graniel C, Frias Mendivil M, Chanona Vilchis G, Gomez C, De La Garza Salazar J. Significance of microvascular density (MVD) in cervical cancer recurrence. Int J Gynecol Cancer. 2003;13(6):856-62.

10. Cooper RA, Wilks DP, Logue JP, Davidson SE, Hunter RD, Roberts SA West CM. High tumor angiogenesis is associated with poorer survival in carcinoma of the cervix treated with radiotherapy. Clin Cancer Res. 1998;4(11):2795-800

11. Lee JS, Choi YD, Lee JH, Nam JH, Choi C, Lee MC, Park CS, Kim HS, Min KW. Expression of PTEN in the progression of cervical neoplasia and its relation to tumor behavior and angiogenesis in invasive squamous cell carcinoma. J Surg Oncol. 2006;93(3):233-40.

12. Petsuksiri J, Chuangsuwanich T, Pattaranutaporn P, Kanngurn S. Angiogenesis in stage IIIB squamous cell carcinoma of uterine cervix: reproducibility of measurement and preliminary outcome as a prognostic factor. J Med Assoc Thai. 2004;87(7):794-9.

13. Dai C, Wang M, Lu J, Dai Z, Lin S, Yang P, Tian T, Liu X, Min W, Dai Z. Prognostic and predictive values of PD-L1 expression in patients with digestive system cancer: a meta-analysis. OncoTargets Ther. 2017;10:3625-34.

14. Parmar MK, Torri V, Stewart L. Extracting summary statistics to perform meta-analyses of the published literature for survival endpoints. Stat Med. 1998;17(24):2815-34

15. Higgins JP, Thompson SG, Deeks JJ, Altman DG. Measuring inconsistency in meta-analyses. BMJ. 2003;327(7414):557-60.

16. Sterne JA, Egger M, Smith GD. Systematic reviews in health care: investigating and dealing with publication and other biases in meta-analysis. BMJ. 2001:323(7304):101-5.

17. Dellas A, Moch H, Schultheiss E, Feichter G, Almendral A, Gudat F, Torhorst J. Association of tumor induced vascularization with clinicopathological parameters in cervical neoplasm. Int J Oncol. 1997;11(1):105-9.

18. Hockel S, Schlenger K, Vaupel P, Hockel M. Association between host tissue vascularity and the prognostically relevant tumor vascularity in human cervical cancer. Int J Oncol. 2001;19(4):827-32.

19. Kaku T, Hirakawa T, Kamura T, Amada S, Kinukawa N, Kobayashi H, Sakai K, Ariyoshi K, Sonoda K, Nakano H. Angiogenesis in adenocarcinoma of the uterine cervix. Cancer. 1998:83(7):1384-90.

20. Moriyama S, Kotera K, Khan KN, Sato F, So Y, Fujishita A, Matsuda K, Nakajima $\mathrm{H}$, Ishimaru TEA. Prognostic significance of tumor volume and microvessel density in squamous cell carcinoma of uterine cervix. Acta Med Nagasakiensia. 2009:53(4):77-84

21. Randall LM, Monk BJ, Darcy KM, Tian C, Burger RA, Liao SY, Peters WA, Stock RJ, Fruehauf JP. Markers of angiogenesis in high-risk, early-stage cervical cancer: a Gynecologic Oncology Group study. Gynecol Oncol. 2009;112(3):583-9.

22. Schlenger K, Hockel M, Mitze M, Schaffer U, Weikel W, Knapstein PG, Lambert A. Tumor vascularity - a novel prognostic factor in advanced cervical carcinoma. Gynecol Oncol. 1995;59(1):57-66.

23. Zijlmans HJ, Fleuren GJ, Hazelbag S, Sier CF, Dreef EJ, Kenter GG, Gorter A. Expression of endoglin (CD105) in cervical cancer. Br J Cancer. 2009;100(10):1617-26.

24. van der Veldt AA, Hooft L, van Diest PJ, Berkhof J, Buist MR, Comans EF, Hoekstra OS, Molthoff CF. Microvessel density and p53 in detecting cervical cancer by FDG PET in cases of suspected recurrence. Eur J Nucl Med Mol Imaging. 2006;33(12):1408-16.

25. Tjalma W, Van Marck E, Weyler J, Dirix L, Van Daele A, Goovaerts G, Albertyn G, van Dam P. Quantification and prognostic relevance of angiogenic parameters in invasive cervical cancer. Br J Cancer. 1998;78(2):170-4.

26. Obermair A, Wanner C, Bilgi S, Speiser P, Kaider A, Reinthaller A Leodolter S, Gitsch G. Tumor angiogenesis in stage IB cervical cancer: correlation of microvessel density with survival. Am J Obstet Gynecol. 1998;178(2):314-9.

27. Uzzan B, Nicolas P, Cucherat M, Perret GY. Microvessel density as a prognostic factor in women with breast cancer: a systematic review of the literature and meta-analysis. Cancer Res. 2004;64(9):2941-55.

28. Yu M, Liu L, Liang C, Li P, Ma X, Zhang Q, Wei Y. Intratumoral vessel density as prognostic factors in head and neck squamous cell carcinoma: a metaanalysis of literature. Head Neck. 2014;36(4):596-602. 
29. Nowak A, Grzegrzolka J, Paprocka M, Piotrowska A, Rys J, Matkowski R, Dziegiel P. Nestin-positive microvessel density is an independent prognostic factor in breast cancer. Int J Oncol. 2017;51 (2):668-76.

30. Barbu I, Craitoiu S, Simionescu CE, Dragnei AM, Margaritescu C. CD105 microvessels density, VEGF, EGFR-1 and c-erbB-2 and their prognostic correlation in different subtypes of cervical adenocarcinoma. Rom J Morphol Embryol. 2013;54(3):519-30.
31. Vieira SC, Silva BB, Pinto GA, Vassallo J, Moraes NG, Santana JO, Santos LG, Carvasan GA, Zeferino LC. CD34 as a marker for evaluating angiogenesis in cervical cancer. Pathol Res Pract. 2005;201(4):313-8.

32. Tanigawa N, Amaya H, Matsumura M, Shimomatsuya T, Horiuchi T, Muraoka R, Iki M. Extent of tumor vascularization correlates with prognosis and hematogenous metastasis in gastric carcinomas. Cancer Res. 1996;56(11):2671-6.
Ready to submit your research? Choose BMC and benefit from:

- fast, convenient online submission

- thorough peer review by experienced researchers in your field

- rapid publication on acceptance

- support for research data, including large and complex data types

- gold Open Access which fosters wider collaboration and increased citations

- maximum visibility for your research: over $100 \mathrm{M}$ website views per year

At BMC, research is always in progress.

Learn more biomedcentral.com/submissions 